\title{
IZw18, or The Picture of Dorian Gray: The More You Watch it, The Older it Gets
}

\author{
M. Tosi ${ }^{1}$, A. Aloisi ${ }^{2}$, J. Mack ${ }^{3}$, and M. Maio ${ }^{1}$ \\ ${ }^{1}$ INAF - Osservatorio Astronomico di Bologna, Bologna, Italy \\ email:monica.tosi@oabo.inaf.it \\ ${ }^{2}$ ESA and Space Telescope Science Institute, Baltimore, USA \\ ${ }^{3}$ Space Telescope Science Institute, Baltimore, USA
}

\begin{abstract}
IZw18 has been recurrently claimed to be a young galaxy, but stars of increasingly older ages are found every time deeper magnitude levels are reached with high-resolution photometry: from the original few Myrs to, possibly, several Gyrs. We summarize the history of IZw18's age and an HST project which will allow us to derive both its distance and age.
\end{abstract}

Keywords. galaxies: evolution, galaxies: stellar content, galaxies: individual (IZw18).

\section{Introduction}

The Blue Compact Dwarf galaxy IZw18 is the most metal poor star-forming galaxy ever discovered, with a metallicity between $1 / 30$ and $1 / 50$ of solar, depending on the adopted solar value (e.g., Searle \& Sargent 1972, Izotov \& Thuan 2004). IZw18 is not only extremely metal poor, but also very blue and full of gas, three properties that originally suggested it to be forming now its first stars and make it the system in the local Universe most similar to primeval galaxies. Soon after its discovery, the basic question became whether IZw18 is so metal poor because a) it has started so recently to form stars that they haven't had time to significantly pollute the medium, or b) because its star formation (SF) activity, although occurring over a long period of time, has proceeded at a rate too low for an efficient chemical enrichment, or c) because strong galactic winds removing from the system most of the metals have accompanied a normal SF activity. More than thirty years later, its age and distance are still subjects of hot debates.

The safest way to estimate the age of nearby galaxies is from the Colour-Magnitude Diagram (CMD) of their resolved stellar populations, but for IZw18, at 10-20 Mpc, this is feasible only with HST and yet challenging. Our novel starts with the advent of HST.

\section{IZw18 looks older everytime we watch it}

The first CMDs derived from HST-WFPC2 images (Hunter \& Thronson 1995) seemed to confirm that the stars in IZw18 are only a few 10 Myr old, until Aloisi, Tosi \& Greggio (1999) identified a number of faint red stars falling in the Asymptotic Giant Branch (AGB) region of the CMD, if not on the RGB (Fig. 1(a)). A result implying that stars at least 500 Myr old are present in the galaxy, and confirmed by Ostlin (2000), who independently measured the same AGB stars on near-infrared HST-Nicmos images. When the ACS became available on HST, with much higher sensitivity, Izotov \& Thuan (2004, hereafter IT04) reobserved IZw18 and confirmed the existence of the AGB, but claimed that no star on the RGB could be detected, thus putting an upper limit of 500 Myr to the age of the system. However, when other people have reduced the same ACS images, they have reached much fainter magnitudes than IT04. The CMD derived by IT04 

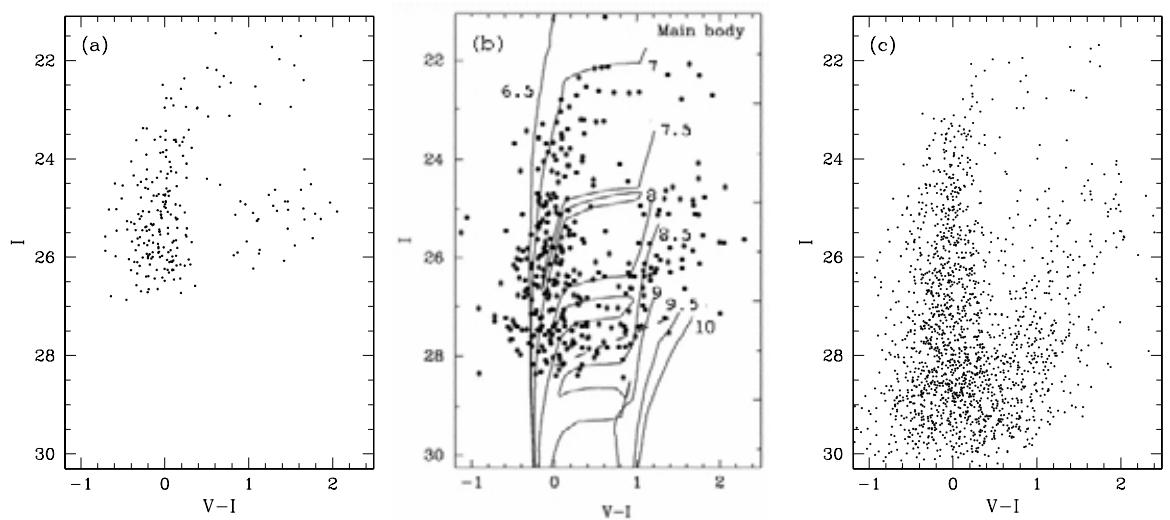

Figure 1. CMDs of the stars resolved in the Main Body of IZw18: (a) by Aloisi et al. (1999) from WFPC2, (b) by IT04 from ACS, $(c)$ by us from the same ACS images as IT04.

from their HST-ACS images is shown in Fig. $1(b)$ together with theoretical isochrones of the labelled $\log ($ age $)$ and assuming a distance of $15 \mathrm{Mpc}$. The CMD obtained by us from the same data is shown in Fig. $1(c)$. Momany et al. (2005) also obtained a CMD like ours, more than two mags deeper than IT04's. It is striking that IT04's photometry stops just where the tip of the RGB is expected ( $\mathrm{I} \simeq 27.2$ at $15 \mathrm{Mpc}$ ), as indicated by the two redder isochrones. We believe that the increase in number of stars occurring in our CMD just at $\mathrm{I} \simeq 27.2$ despite the increasing level of incompleteness is strongly suggestive of the presence of the RGB. As shown by the isochrone labels in Fig. 1(b), this would imply an age significantly older than 2 Gyr, possibly 10 Gyr or more. However, this indication cannot be confirmed until better estimates of the galaxy distance are available to precisely constrain the expected magnitude of the RGB tip.

This is why we have re-observed IZw18 for 24 HST-ACS orbits at 12 different epochs (prop. 10586, PI Aloisi) to perform time series photometry, identify Cepheids, measure their light curves and infer their distance from the period-luminosity and periodluminosity-colour relations, following the method applied in the $\mathrm{HST} \mathrm{H}_{0}$ key-project (Saha et al. 2006 and references therein). To this aim we are also computing non-linear convective pulsation models for the metallicity of IZw18, which will be used for safer light curve analyses and distance derivation, as done for RR Lyraes in the LMC by Marconi \& Clementini (2005). The combination of this new photometry with the archival data will allow us to derive deeper CMDs to better search for RGB stars. Independent distance estimates will be inferred from the brightness of the Carbon stars and of the RGB tip (if any). We have already identified $\sim 30$ variables and the data analysis is in progress: stay tuned on the IZw18 age serial!

\section{References}

Aloisi, A., Tosi, M., \& Greggio, L. 1999, AJ 118, 302

Hunter, D.A. \& Thronson, H.A. 1995, ApJ 452, 238

Izotov, Y.I. \& Thuan, T.X. 2004, ApJ 616, 768

Marconi, M. \& Clementini, G. 2005, AJ 129, 2257

Momany, Y., Held, E.V., Saviane, I., Bedin, L.R., Gullieuszik, M., Clemens, M., Rizzi, L., Rich, M.R., \& Kuijken, K. 2005, A\&A 439, 111

Ostlin, G. 2000, ApJ (Letters) 535, L99

Saha, A., Thim, F., Tammann, G. A., Reindl, B., \& Sandage, A. 2006, ApJS 165, 108

Searle, L. \& Sargent, W.L.W. 1972, ApJ 173,25 